\title{
Аналитическая модель случайного распределения объектов двух сортов на двумерной плоскости с гексагональной структурой
}

\author{
Прудковский А.Г. \\ Институт геохимии и аналитической химии им. В.И. Вернадского Российской Академии наук, \\ Москва
}

Поступила в редакцию 25.09.2019 г.

DOI: $10.17308 /$ sorpchrom.2019.19/1177

Данная работа посвящена исследованию равновесных состояний абстрактных двухфазных систем с учётом симметрий на примере сорбции объектов двух сортов на гексагональную плоскость. В первом приближении подобные процессы управляются некоторым коэффициентом распределения, который легко может быть измерен на опыте. Однако определение вероятности образования при этом конгломератов однотипных объектов так просто определить невозможно. В данной работе приведено точное решение данной вероятностной задачи, а также обсуждены вопросы нарушения законов статистики, которые могут происходить в результате взаимодействия сорбируемых объектов.

Предложенная методика построения формул легко может быть обобщена на иные симметричные структуры, отличные от гексагональных, а также на иную структуру центров сорбции, отличных от плоскости. Результаты работы могут быть применены для исследования реакций обмена в задачах адсорбции и химического равновесия при наличии тех или иных симметрий в сочетании с вероятностным распределением.

Ключевые слова: математическое моделирование, симметрия, случайность, распределение, реакции обмена.

\section{An analytical model of the random distribution of two varieties objects of on a two-dimensional plane with a hexagonal structure}

\author{
Prudkovskii A.G. \\ Vernadsky Institute of Geochemistry and Analytical Chemistry of Russian Academy of Sciences \\ (GEOKHI RAS). Moscow
}

In a first approximation, sorption processes are characterized by equilibrium isotherms with constant or variable coefficients. But the equilibrium coefficients reflect only the amount of the sorbed component, but not the geometry of its distribution in the sorbent phase. A cluster analysis of the distribution of objects during their sorption can lead to completely unexpected results. Moreover, as shown in this paper, the emergence and decomposition of clusters of various adsorbate objects does not affect the current equilibrium coefficient, it is a low-energy process that can very well be carried out using the thermal energy of the environment. Thus, by manipulating the probabilities of the formation and destruction of clusters, it is theoretically possible to achieve very interesting effects up to the creation of vibrational reactions of the creation and decomposition of clusters. Although the implementation of all these theoretical model projects is a matter for chemists. Not everything that is real in theory can be put into practice. It should also be noted that this problem is closely intertwined with the theory of percolation, where the conditions for the appearance of infinitely 
long clusters with a random distribution of defects in space are also sought. In the future, a more detailed study of all these effects is expected.

So, for modeling, we chose the simplest hexagonal surface structure. We assume that the sorption isotherm is known to us from experience. Specific experimental measurements of the sorption isotherm have already been carried out in our laboratory and published earlier. Our goal is to conduct a cluster analysis of the adsorbate using the solution of the model problem and highlight the most interesting patterns that could subsequently be confirmed by microscopic studies. Our earlier numerical experiments showed the possibility of clusters of objects of the same type during their sorption. In this paper, we give a mathematical introduction to the problems of this problem using the example of studying the equilibrium states of abstract twophase systems during the sorption of objects of two kinds on the hexagonal plane. Then the issues of possible violation of a simple probabilistic picture in the presence of interaction of sorbed objects are discussed. This is all the more important because in such conglomerates certain interactions between similar objects are possible, which can violate a simple probabilistic picture. In this regard, the issues of violation of the laws of statistics that can occur as a result of the interaction of adsorbed objects that can violate the exact solution of the probabilistic problem are discussed here, and methods for modifying the formulas in this case are proposed.

Conclusion: It should be noted that the proposed method for constructing formulas can easily be generalized to other symmetric structures other than hexagonal, as well as to a different structure of sorption centers other than the plane. The results of the work can be used to study exchange reactions in the problems of adsorption and chemical equilibrium in the presence of certain symmetries in combination with a probability distribution, as well as to predict and design new reactions with a theoretically predicted result.

Keywords: mathematical modeling, symmetry, randomness, distribution, exchange reactions.

\section{Введение}

Данная работа посвящена чисто математической проблеме, хотя модели, аналогичные построенной здесь, могут иметь реальное применение в некоторых задачах химического обмена. При моделировании процессов в многокомпонентных системах часто возникают проблемы статистического распределения объектов, причём с сохранением некоторых симметрий, определяемых условиями задачи. В данной работе показано решение этих проблем на примере модельной задачи о случайном распределении объектов двух сортов на плоскости с гексагональной структурой. Ранее нами были проведены модельные эксперименты, описывающие возможные эффекты, связанные с возникновением крупных кластеров однотипных объектов при их сорбции [1]. При этом нами использовалась опытные изотермы сорбции из работ [2, 3]. Кроме построения точного решения задачи, в данной работе рассмотрены и некоторые возможные варианты распределений при возможных нарушениях вероятностных законов, связанных с взаимодействием сорбированных объектов.

\section{Теоретическая часть}

Итак, имеем трёхмерное пространство, заполненное объектами двух сортов: $c_{1}, c_{2}$ - доли или вероятности этих объектов в пространстве $c_{1}+c_{2}=1$. Кроме того, пусть имеется бесконечная плоскость с гексагональной структурой, на которой в статистическом равновесии с пространством также находятся те же объекты двух сортов: их доли обозначим через $a_{1}, a_{2} ; a_{1}+a_{2}=1$. Гексагональная структура позволяет каждому объекту на плоскости иметь по шесть соседей рис.1 . Доли объектов на плоскости $a_{1}, a_{2}$ связаны с долями тех же объектов в пространстве уравнением:

$$
\frac{a_{1}}{c_{1}}=K\left(c_{1}\right) \frac{a_{2}}{c_{2}}
$$

где $K\left(c_{1}\right)$ - некоторая функция, связывающая распределение объектов в пространстве с распределением их же на плоскости. В реальных задачах функция $K\left(c_{1}\right)$ может 
быть получена из опыта, однако, опытным путём невозможно получить среднее число соседей того же типа у каждого из объектов.

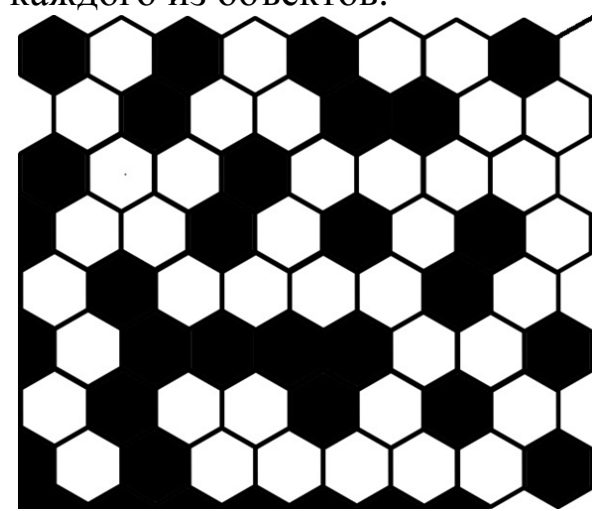

Рис. 1 Фрагмент плоскости с некоторым случайным распределением объектов двух сортов.

В простейшем случае $K\left(c_{1}\right) \equiv 1$ задача решается стандартными формулами теории вероятности [4].

Обозначим через $a_{10}$ - долю одиночных объектов первого типа на плоскости, а через $a_{11}, a_{12}, a_{13}, a_{14}, a_{15}, a_{16}$ доли объектов первого типа, имеющих соответственно одного, двух, и так далее - до шести соседей того же типа. Аналогично через $a_{20}, a_{21}, a_{22}, a_{23}, a_{24}, a_{25}, a_{26}$ - обозначим доли объектов второго типа на плоскости, имеющих соответственно от нуля до шести соседей своего типа. Таким образом, имеем:

$$
\begin{aligned}
& a_{10}+a_{11}+a_{12}+a_{13}+a_{14}+a_{15}+a_{16}=a_{1} \\
& a_{20}+a_{21}+a_{22}+a_{23}+a_{24}+a_{25}+a_{26}=a_{2}
\end{aligned}
$$

В соответствии со стандартными формулами вероятностного распределения имеем:

$$
\begin{gathered}
a_{1 m}=c_{1} C_{6}^{m} c_{1}^{m}\left(1-c_{1}\right)^{6-m} ; a_{2 m}=c_{2} C_{6}^{m} c_{2}^{m}\left(1-c_{2}\right)^{6-m} \\
a_{1}=\sum_{m=0}^{6} a_{1 m}=c_{1}\left(c_{1}+c_{2}\right)^{6}=c_{1} ; \quad a_{2}=\sum_{m=0}^{6} a_{2 m}=c_{2}\left(c_{1}+c_{2}\right)^{6}=c_{2} ; K\left(c_{1}\right)=\frac{a_{1} c_{2}}{a_{2} c_{1}} \equiv 1
\end{gathered}
$$

В случае константы $K\left(c_{1}\right)=K_{0} \neq 1$ также можно воспользоваться вероятностными формулами, представив некое формальное сито, которое пропускает к плоскости только некие доли $q_{1}\left(c_{1}\right), q_{2}\left(c_{2}\right)$ всех объектов внешнего пространства в соответствии с правилами:

$$
q_{1}\left(c_{1}\right)+q_{2}\left(c_{2}\right)=1 ; \frac{q_{1}\left(c_{1}\right)}{q_{2}\left(c_{2}\right)}=K_{0}
$$

Решение системы уравнений (4):

$$
q_{1}\left(c_{1}\right)=\frac{c_{1}}{c_{1}+K_{0}^{-1} c_{2}}=\frac{c_{1} K_{0}}{K_{0} c_{1}+c_{2}}, q_{2}\left(c_{2}\right)=\frac{c_{2}}{K_{0} c_{1}+c_{2}}=\frac{c_{2} K_{0}^{-1}}{c_{1}+K_{0}^{-1} c_{2}},
$$

а доли объектов с разным количеством соседей можно найти по формулам, аналогичным формулам (3):

$$
a_{1 m}=q_{1} C_{6}^{m} q_{1}^{m}\left(1-q_{1}\right)^{6-m} ; a_{2 m}=q_{2} C_{6}^{m} q_{2}^{m}\left(1-q_{2}\right)^{6-m}
$$

откуда следует:

$$
a_{1}=\sum_{m=0}^{6} a_{1 m}=q_{1}\left(q_{1}+q_{2}\right)^{6}=q_{1} \quad a_{2}=\sum_{m=0}^{6} a_{2 m}=q_{2}\left(q_{1}+q_{2}\right)^{6}=q_{2}
$$




$$
K\left(c_{1}\right)=\frac{a_{1} c_{2}}{a_{2} c_{1}}=\frac{q_{1} c_{2}}{q_{2} c_{1}}=K_{0}
$$

Рассмотрим теперь случай, когда величина $K\left(c_{1}\right)$ непостоянна. В рамках чистой статистики можно решать эту задачу чисто формально, подставив в формулы $(4,7)$ величину $K\left(c_{1}\right)$, вместо $K_{0}$. Однако, если непостоянство величины $K\left(c_{1}\right)$ связано с взаимодействиями между соседними объектами на плоскости, то формулы статистики могут быть нарушены и корректность формул $(6,7)-$ под вопросом. В этом случае решение задачи о долях объектов с разным количеством соседей может иметь не единственное решение. Действительно, введём произвольную положительную функцию $\widetilde{K}\left(c_{1}\right)$ и соответствующие ей доли:

$$
\tilde{q}_{1}\left(c_{1}\right)=\frac{c_{1} \tilde{K}\left(c_{1}\right)}{c_{1} \tilde{K}\left(c_{1}\right)+c_{2}}, \tilde{q}_{2}\left(c_{2}\right)=\frac{c_{2}}{c_{1} \tilde{K}\left(c_{1}\right)+c_{2}}, \tilde{q}_{1}+\tilde{q}_{2}=1
$$

Теперь с помощью наших произвольно заданных функций (8) можно также произвольно задать и доли объектов на плоскости:

$$
\tilde{a}_{1 m}=q_{1} C_{6}^{m} \tilde{q}_{1}^{m}\left(1-\tilde{q}_{1}\right)^{6-m} ; \tilde{a}_{2 m}=q_{2} C_{6}^{m} \tilde{q}_{2}^{m}\left(1-\tilde{q}_{2}\right)^{6-m},
$$

где величины $q_{i}=q_{i}\left(c_{i}\right)$ вычисляются по формулам (5), а $\tilde{q}_{i}=\tilde{q}_{i}\left(c_{i}\right)$ - по произвольным формулам (8).

Заметим, что такое произвольное задание долей в формуле (8) никак не сказывается на коэффициенте $K\left(c_{1}\right)$. Действительно, по формулам (9) имеем:

$$
\begin{gathered}
a_{1}=\sum_{m=0}^{6} \tilde{a}_{1 m}=q_{1}\left(\tilde{q}_{1}+\tilde{q}_{2}\right)^{6}=q_{1} \quad a_{2}=\sum_{m=0}^{6} \tilde{a}_{2 m}=q_{2}\left(\tilde{q}_{1}+\tilde{q}_{2}\right)^{6}=q_{2} ; \\
\frac{a_{1} c_{2}}{a_{2} c_{1}}=\frac{q_{1} c_{2}}{q_{2} c_{1}}=K\left(c_{1}\right)
\end{gathered}
$$

Таким образом, экспериментальное измерение зависимости $K\left(c_{1}\right)$ ещё не даёт достаточно информации о распределении объектов по долям с определённым количеством соседей данного вида. Однако, при наличии дополнительной информации формулы (5-10) можно модифицировать в соответствии с этой информацией.

Пример 1. Предположим, что парное взаимодействие между объектами одного вида повышает (или понижает) вероятность их появления в $\beta$ раз, тогда из уравнения для вероятности возникновения пары $\tilde{a}_{11}$, найдём:

$$
\begin{aligned}
\tilde{a}_{11}=q_{1} C_{6}^{1} \tilde{q}_{1}^{1}\left(1-\tilde{q}_{1}\right)^{6-1} & =\beta q_{1} C_{6}^{1} q_{1}^{1}\left(1-q_{1}\right)^{6-1} ; \Rightarrow \tilde{q}_{1}^{1}\left(1-\tilde{q}_{1}\right)^{6-1}=\beta q_{1}^{1}\left(1-q_{1}\right)^{6-1} \\
& \Rightarrow \frac{\tilde{K}\left(c_{1}\right)}{\left(c_{1} \tilde{K}\left(c_{1}\right)+c_{2}\right)^{6}}=\beta \frac{K\left(c_{1}\right)}{\left(c_{1} K\left(c_{1}\right)+c_{2}\right)^{6}}
\end{aligned}
$$

Нелинейное уравнение (11) позволяет вычислить величину $\widetilde{K}\left(c_{1}\right)$ через остальные уже известные величины, затем подставить её в формулы (8-10), получив, таким образом, все искомые распределения. Следует, однако, учесть, что приближение (11) работает только при малых значениях $c_{1}$, когда вариантами с большим количеством соседей можно пренебречь.

Пример 2. Предположим, что между объектами первого типа есть некоторое взаимодействие, в то время, как объекты второго типа не взаимодействуют друг с другом. Тогда можно предположить, что все распределения объектов первого типа полностью определяются величиной $K(1)$, величины $\tilde{a}_{i j}$ в этом случае можно оценить по формуле (9), подставив в неё: 


$$
\tilde{q}_{1}\left(c_{1}\right)=\frac{c_{1} K(1)}{c_{1} K(1)+c_{2}}, \quad \tilde{q}_{2}\left(c_{2}\right)=\frac{c_{2}}{c_{1} K(1)+c_{2}}
$$

\section{Обсуждение результатов}

Заметим, что описанная здесь модель легко обобщается на любое другое количество соседей на плоскости, отличных от шести, а саму плоскость вполне можно заменить, некими центрами притяжения, плавающими в том же пространстве. Заметим также, что последнее дополнение позволяет вводить в развитую здесь теорию произвольные дополнительные вариации, связанные с особыми свойствами взаимодействующих объектов на плоскости, нарушающих вероятностное описание процесca.

\section{Заключение}

Представленная здесь работа достаточно абстрактна и не имеет прямого практического применения, однако она касается принципов моделирования процессов обмена в химии при наличии симметрий относительно типа взаимодействующих молекул и вполне может быть полезна при создании аналогичных моделей равновесных распределений в реальных задачах. Отметим также, что модели, рассмотренные здесь, могут быть полезны и в теории перколяции [5], где также оценивается величина кластеров вероятностных объектов, представляющих дефекты структуры.

\section{Список литературы}

1. Хамизов Р.Х., Прудковский А.Г. // Тезисы докладов VII Всероссийского симпозиума $и$ Школь-конференции молодых ученьх, посвященных 115-летию со дня открытти хроматографии русским учёным М.С. Цветом. 2018. С. 67-68.

2. Krachak A.N., Nikashina V.A., Ksenzenko V.I. // Bulletin of the Academy of Sciences of the USSR. Division of Chemical Sciences. 1983. Vol. 32. No 1. pp. 162-164.

\section{References}

1. Hamizov R.H., Prudkovskij A.G., Tezisy dokladov VII Vserossijskogo simpoziuma i Shkolykonferencii molodyh uchenyh, posvjashhennyh 115-letiju so dnja otkrytija hromatografii russkim uchjonym M.S. Cvetom6 2018, pp. 67-68.

2. Krachak A.N., Nikashina V.A., Ksenzenko V.I., Bulletin of the Academy of Sciences of the USSR. Division of Chemical Sciences, 1983, Vol. 32, No 1, pp. 162-164.

Прудковский Андрей Гаральдович - д.фм.н., ведущий научный сотрудник, лаборатория сорбционных методов, ГЕОХИ РАН, Москва
3. Ножов А.М., Кособрюхова О.М., Хамизов P.Х. // Сорбиионные и хроматографические проиессы. 2003. Т. 3. № 2. С. 159-168.

4. Ширяев А.Н. Вероятность 1. Элементарная теория вероятностей. Математические основания. Предельные теоремы. М. МЦНМО. 2007. 552 .

5. Меньшиков М.В., Молчанов С.А., Сидоренко А.Ф. // Итоги науки и техн. Сер. Теор. вероятн. Мат. стат. Теор. кибернет. 24. ВИНИТИ. М. 1986. С. 53-110.

3. Nozhov A.M., Kosobrjuhova O.M., Hamizov R.H., Sorbtsionnye i khromatograficheskie protsessy, 2003, Vol. 3, No 2, pp. 159-168.

4. Shirjaev A.N., Verojatnost' 1. Jelementarnaja teorija verojatnostej. Matematicheskie osnovanija. Predel'nye teoremy, M., MCNMO, 2007, 552 p.

5. Men'shikov M.V., Molchanov S.A., Sidorenko, A.F., J. Soviet Math., 1988, Vol. 42, No 4, pp. 1766-1810.

Prudkovskii Andrei G. - Ph.D. (Phys.Math.), Senior researcher, Lab of Sorption Methods, GEOKHI RAS, Moscow, prudkovsky@gmail.com 